\title{
Teaching Creative Interface Design: Possibilities and Pitfalls
}

\author{
Janet Wesson \\ Nelson Mandela Metropolitan University \\ PO Box 77000 \\ Port Elizabeth 6031, SOUTH AFRICA \\ $+27415042323$ \\ janet.wesson@nmmu.ac.za \\ WWW home page: http://www.nmmu.ac.za
}

\begin{abstract}
Interface design is an essential aspect of any interactive system and thus a core component of most Human-Computer Interaction (HCI) curricula. Teaching creative interface design is, however, a challenging task, as it involves both an understanding of HCI theory and practice. A trade-off exists between enforcing the use of standard design aids such as guidelines and patterns, or encouraging the development of creative design solutions. This paper discusses the tensions that exist between these two approaches and explores the possibilities for developing a combined approach to teaching creative interface design. This approach, called 'Usable Creativity' aims to produce usable and creative design solutions.
\end{abstract}

\section{Introduction}

Creating good interfaces requires designers to think really hard about how they want to visually represent the interface. In essence, interface design comprises designing the external (visual) representation; determining how data is visualised (information visualisation and visual properties); choosing an appropriate design metaphor; and providing effective interaction techniques.

This paper firstly discusses how design guidelines and patterns can be used to support the design process and how design metaphors can be used to assist users with the learning process. Several different approaches to teaching creative design are then reviewed. Finally, a combined approach for teaching creative interface design is presented together with some student examples developed using this approach. 


\section{Design Guidelines and Patterns}

Design guidelines are a commonly used and generally accepted aid for interface design [1]. Guidelines specify what to provide and what not to provide in the interface and are intended to help designers explain and improve the interface design [2]. Design guidelines are typically derived from a mix of theory-based knowledge, experience and common-sense. Examples of such guidelines include Shneiderman's eight golden rules for interface design [3]:

1. Strive for consistency.

2. Enable frequent users to use shortcuts.

3. Offer informative feedback.

4. Design dialogues to yield closure.

5. Provide error prevention and simple error handling.

6. Permit easy reversal of actions.

7. Support internal locus of control.

Usability principles (heuristics) are similar to design guidelines, except more prescriptive, and are used mainly as a basis for the heuristic evaluation of interactive systems. These include Nielsen's ten heuristics [4] as well as others for designing specific types of systems, such as IBM's web design guidelines [5] and Gong and Taresewich's guidelines for mobile interface design [6] .

Interaction design patterns have been proposed as alternatives to design guidelines [7]. Some evidence exists that interfaces designed using patterns are better than the equivalent interfaces designed using guidelines [8]. More research is needed, however, to determine if there is a significant difference in quality between the designs produced using patterns and the designs produced using guidelines [9].

\section{Design Metaphors}

Choosing an appropriate design metaphor implies designing the visual representation of the interface to be similar to a physical entity but with its own behaviour and properties, for example the desktop metaphor and the search engine. A design metaphor can be based on an activity, an object or a combination of both.

The use of an appropriate metaphor can exploit the users' familiar knowledge, helping them to understand 'the unfamiliar' [1]. Metaphors conjure up the essence of an unfamiliar activity, thereby enabling users to leverage familiar knowledge to understand other aspects of the unfamiliar functionality.

Using design metaphors can have several benefits as they can:

- make learning new systems easier;

- help users understand the underlying conceptual model; and

- be very innovative.

Several problems, however, also exist with metaphors, since they can:

- break conventional and cultural rules;

- constrain designers in the way that they conceptualize a problem space;

- conflict with design principles; 
- limit the users to only understanding the system in terms of the metaphor;

- cause designers to inadvertently use existing bad designs; and

- limit the designer's imagination in creating new paradigms and models.

It is clear that several pitfalls exist with using design metaphors for interface design. However, this does not have to be the case. Provided designers are aware of these pitfalls and endeavour to develop metaphors that effectively combine familiar knowledge with new functionality, many of the above problems can be avoided [1]. The use of analogy as a basis for interface design can also be very innovative and successful, expanding the use of computers to a greater diversity of people.

\section{Prototyping}

Generating alternative designs is an essential aspect of the interaction design process [1]. Considering alternatives and thinking about different perspectives can provide the designer with considerable insight into the problem space. These alternative designs are normally represented in terms of low-fidelity (paper-based) or highfidelity (software) prototypes.

Prototyping requires converting the user requirements and needs into a visual representation (conceptual model) of the system. The basis for designing this model is the set of user tasks that the system will support. Three different aspects need to be determined:

- Which interaction mode(s) should be used? This will depend on the nature of the users' activities, for example exploring and browsing.

- If an appropriate design metaphor exists (Section 3). This requires an understanding of what the system will do and which are the critical tasks.

- Which is the most suitable interaction paradigm? This requires an understanding of the user and the proposed context of use.

Developing a prototype therefore involves addressing these aspects and using appropriate design guidelines (Section 2) and metaphors (Section 3) to produce a design solution which meets the users' requirements.

The process of generating alternative designs is, however, not as straightforward as one might expect, for several reasons [1]:

- Humans generally stick to what they know works.

- Considering alternatives is important to 'break out of the box'.

- Designers are trained to consider alternatives; software developers generally are not.

This raises the question of how do you generate alternative design solutions? Several ideas have been proposed including:

- Flair and creativity: This requires research and synthesis.

- Seek inspiration: The designer can look at similar systems or look at very different systems.

The problem of generating alternative and innovative designs has resulted in research into teaching creative design, which is discussed in the next section. 


\section{Teaching Creative Design}

Several approaches have been developed to encourage and foster design creativity. These include the following:

- BadIdeas, a technique that uses bad or silly ideas to inspire creativity [10]. This approach generates silly or bad ideas to solve a problem rather than aiming directly for good ideas, thus allowing the student designer to derive metrics with which to evaluate and extend these ideas. It is not clear, however, if the suggestions made to remedy these bad ideas would include the use of design guidelines and result in a usable system.

- Worksheets, which are used to capture aspects of a scene or situation [11]. Worksheets can be used to record stories and anecdotes as well as people. The use of Worksheets allows designers to gather and structure information about the problem space, thus enabling them to easily identify if important information is missing. This technique obviously assists in the requirements analysis phase, but does appear to support creative design.

- Rapid Contextual Design, which includes the use of contextual interviews, affinity diagrams, personas, scenarios, visioning and paper prototypes [12]. Although some aspects of this method, especially contextual interviews, can prove very useful, it is not clear how it encourages creative thinking.

- Metaphors, which can be used to understand the nature of human thinking, and thereby lead to opportunities for creating better designs [13]. Using metaphors can, however, have several pitfalls as discussed earlier in Section 3.

There is no doubt that insufficient time is given in HCI curricula to encourage and foster creativity [14]. To address this issue, Beckhaus proposed seven factors which are needed to support teaching creative design [15]. These factors are:

- Formation of a group and a supportive environment.

- Building confidence in creativity.

- Balance of a clear goal with space to evolve.

- Preparation of a task document.

- Motivation.

- Assessment.

- Assistance versus interference.

In general, successful interface design requires balancing constraints and requirements and teaching creative design requires a similar approach. The issue of assessing creativity is, however, not straight forward, as discussed in the next section.

\section{Assessing Creativity}

The issue of assessing creativity is one of the greatest challenges currently facing teachers. While many teachers believe that it is possible to help students be creative, few believe that it is possible to assess creativity effectively [16]. Yet evaluation is considered critical to the idea of creativity. In addition, the issue of evaluating design 
quality is still largely unresolved, which can lead to inaccurate results [17]. Usability is fairly easy to evaluate using standard techniques like heuristic evaluation (Section 2 ), but evaluating design creativity has not been adequately addressed. There is almost universal agreement, however, that understanding a student's creativity depends on his/her ability to understand and explain it. Reflection would thus seem to be a necessary partner to creativity.

Evaluation of creativity can focus on several aspects including the products produced, the process followed or a combination of these [16]. Several characteristics of a creative product (or system) are included in the definition of 'Functional Creativity', which is well known in the domain of Engineering. These characteristics are as follows:

- Novelty (is it original and surprising?).

- Relevance and effectiveness (does it do what it is supposed to do?).

- Elegance (it is a simple and easy to understand solution?).

- Germinal (does it lead to new ideas and/or products?).

Although this definition is typically used to evaluate the creativity of products produced by engineering students, it can also be used to assess the creativity of interface designs produced by HCI students. Three characteristics of this definition are particularly relevant for assessing design creativity, namely Novelty (is it original or innovative), Relevance and effectiveness (does it do what it is supposed to do) and Germinal (does it lead to new metaphors and/or designs). The other characteristic, namely Elegance, is included in the ten usability heuristics as proposed by Nielsen (as Aesthetic and minimalist design) [10].

The next section proposes a combined approach to teaching creative interface design which encourages creativity whilst still ensuring that the design solutions produced comply with standard design principles

\section{The Usable Creativity Approach}

As discussed previously, design guidelines and patterns can be used to assist with interface design. If combined with a user-centred design approach, these design aids can be used to produce usable design solutions. These design aids do not, however, encourage and foster creativity or the generation of new metaphors or alternative designs.

Some of the current approaches to teaching creative design actively promote innovation and creativity. These approaches do not, however, include the use of the design aids discussed in Section 2 and thus do not address the issue of usability.

An approach is needed, therefore, which provides structure whilst encouraging innovation in order to produce usable and creative design solutions. This approach is expressed in the formula for 'Usable Creativity':

where:

$$
\text { Structure + Innovation } \rightarrow \text { Usable Creativity }
$$

- Structure is provided by design aids such as guidelines and patterns (Section 2); and

- Innovation is provided by metaphors and prototyping (Sections 3 and 4). 
This approach to teaching creative design requires exposing students to HCI theory in terms of guidelines and patterns, and HCI practice in terms of generating alternative, creative designs taking due cognisance of these design aids.

This approach also requires most of the seven factors identified by Beckhaus (Section 5) in order to provide a favourable environment for inventivity and creativity. In particular, the students need to work in small groups to encourage collaboration; the requirements and goals of the project must be clearly defined; a task document should be established; the students should be motivated by allowing them to choose an area in which they are interested; and the choice of prototyping tools should be left to the students.

The approach towards 'Usable Creativity' discussed in this section was used to teach a postgraduate course in HCI to 15 students at the Nelson Mandela Metropolitan University in the first semester of 2006. Two examples of the student designs produced are presented and discussed in the next section.

\section{Examples}

The students were asked to select any interactive system and design prototypes for this system, working in groups of 2 or 3 students. The design documentation required to be submitted comprised the following:

- a description of the users' goals;

- the envisaged context of use;

- the functional and non-functional requirements for the system;

- a low-fidelity (paper-based) prototype for the system;

- feedback from informal user testing; and

- a high-fidelity prototype.

The students were required to include sufficient screenshots to show how the key user tasks and goals would be satisfied. The following sections present a description of the development methodology, two example designs submitted by the students and a discussion of how these designs were evaluated.

\subsection{Development Methodology}

The students were required to use the Interaction Design (ID) Model, as proposed by Preece et al. to develop these prototypes [1]. This methodology comprises four main steps, namely:

- identify requirements;

- develop alternative designs;

- build interactive prototypes; and

- evaluate the prototypes.

The key aspects of this methodology are a user-centred approach, identifying usability and user experience goals, and iterative design and evaluation. This methodology does not, however, prescribe any specific interface design method(s). The students were therefore given lectures on the use of design aids such as 
guidelines and patterns, and the use of interface metaphors to support design. The students were also informed that the evaluation of the design of their prototypes would comprise two main aspects, namely usability and creativity. The approach to teaching interface design thus followed the steps outlined in the 'Usable Creativity' approach as discussed in Section 7.

\subsection{Medical Mobile}

Medical Mobile is a PDA/Pocket PC based system designed to replace the currently inadequate practice of doctors relying on pagers or cell phone messages. Currently, pagers or text messages are still often used to notify doctors of medical emergencies or patients that require urgent attention when they are out of the office or hospital. Such devices do little to indicate the exact nature of the situation that requires their attention. Medical Mobile attempts to remedy this situation by providing an interactive system which alerts doctors to situations that require their urgent attention. The system will provide doctors with exact details regarding the priority of the situation and allow them instant access to patient files and medical histories (see Figures 1 and 2). They will also be able to respond to the messages they receive, and thus provide better care for their patients.

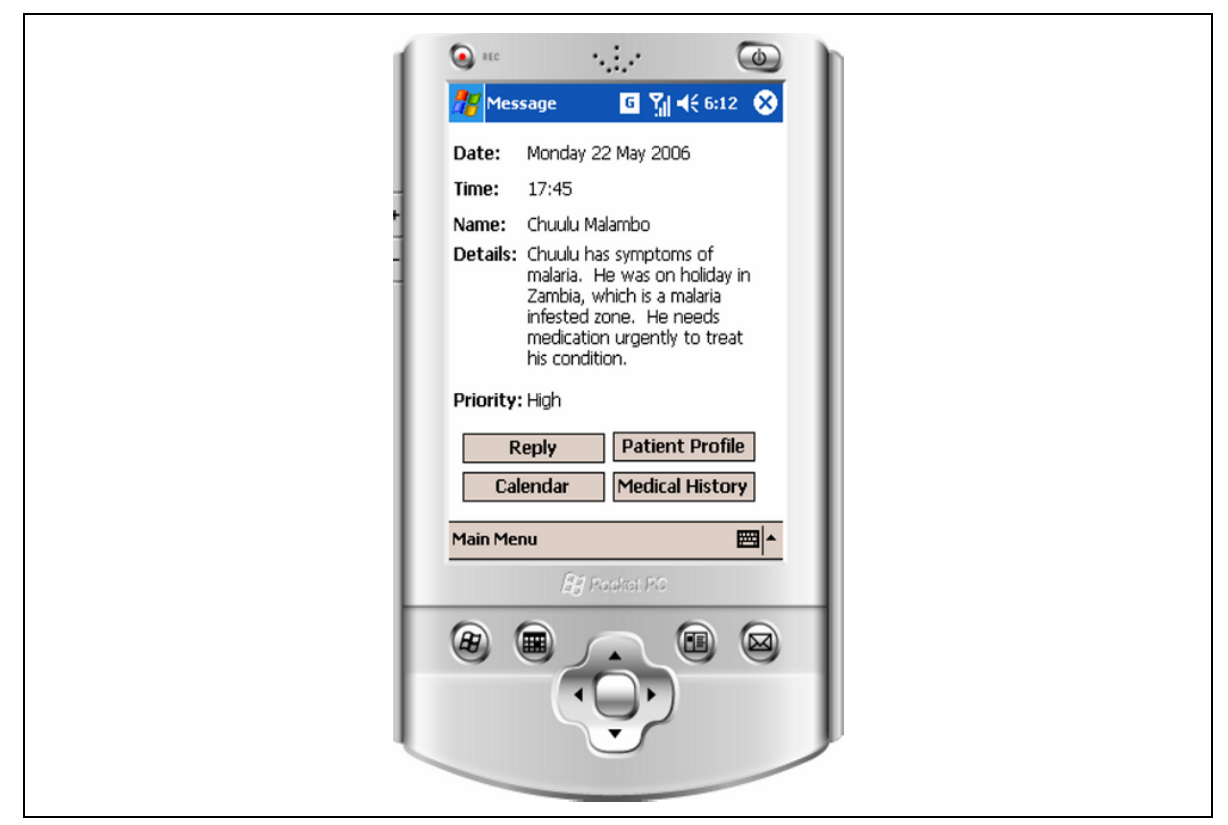

Fig. 1. Message screen in Medical Mobile 


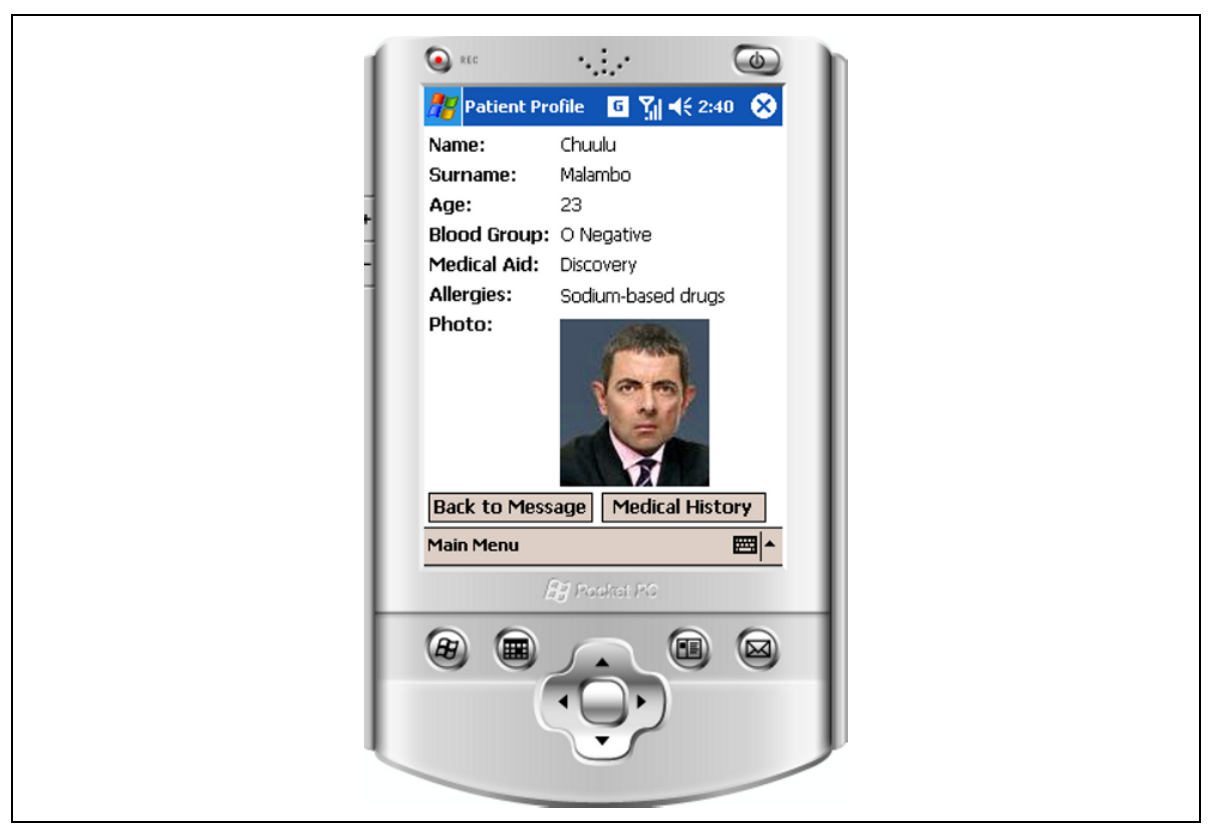

Fig. 2. Patient Profile in Medical Mobile

\subsection{TravelSA}

TravelSA is a PDA based system which allows tourists to obtain information about cities within South Africa and book accommodation in a particular city (Figure 3). Locating areas of interest within a particular city is done using a city map (Figure 4). Users can then view information about a city and the different areas within that city, with the aid of pictures and textual descriptions. Users can also search for accommodation in a selected city based on certain criteria such as price range, accommodation rating and/or distance from the airport (Figure 5). A facility also exists for users to book accommodation and receive confirmation of reservation with a reservation number. 


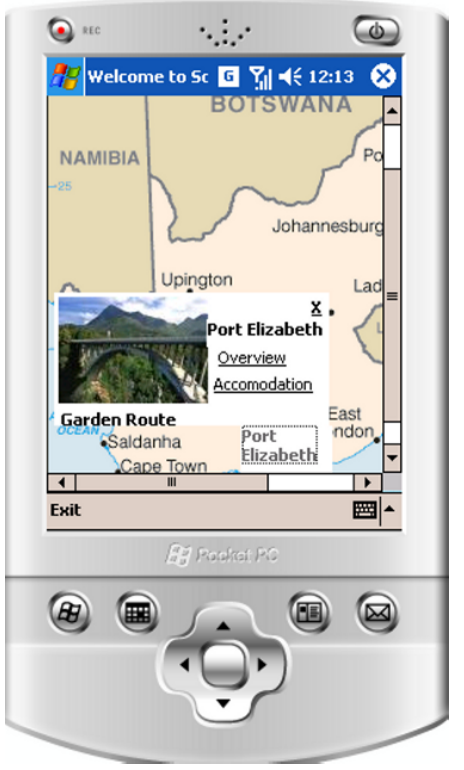

Fig. 3. Selection of a city in TravelSA

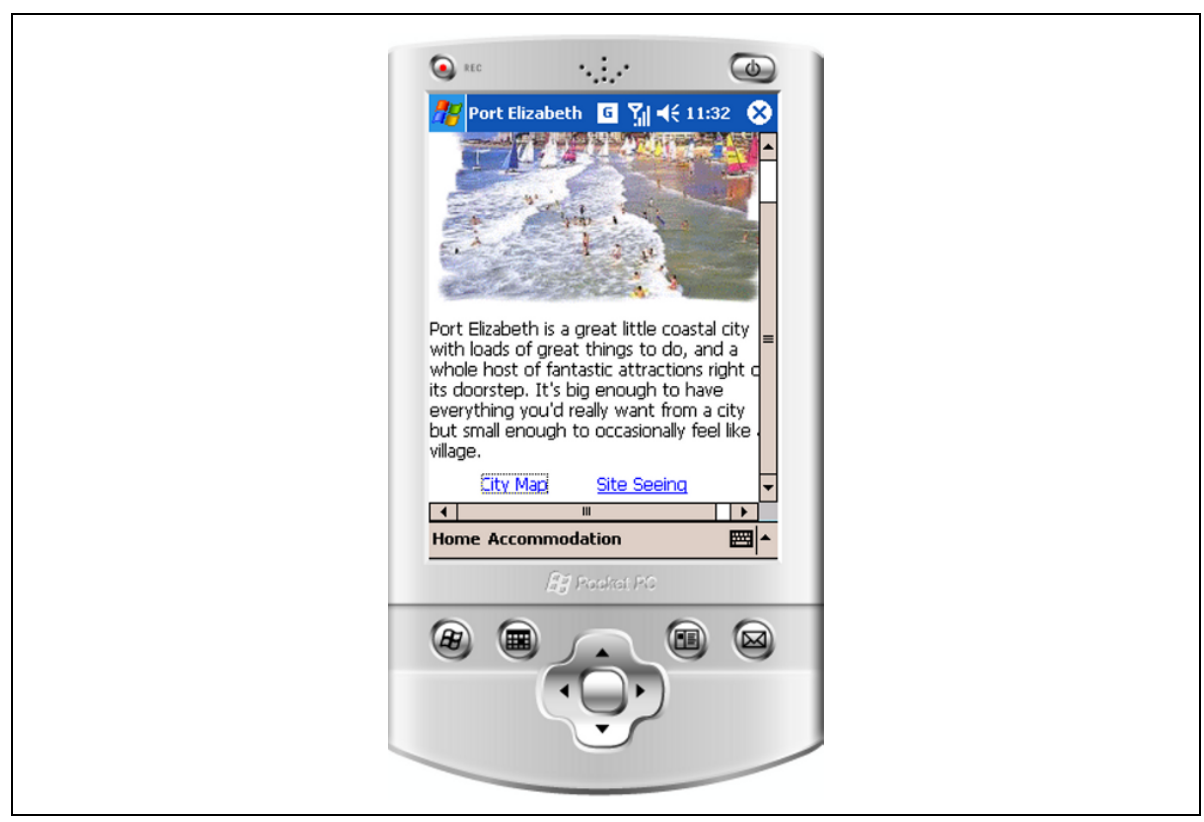

Fig. 4. City information in TravelSA 


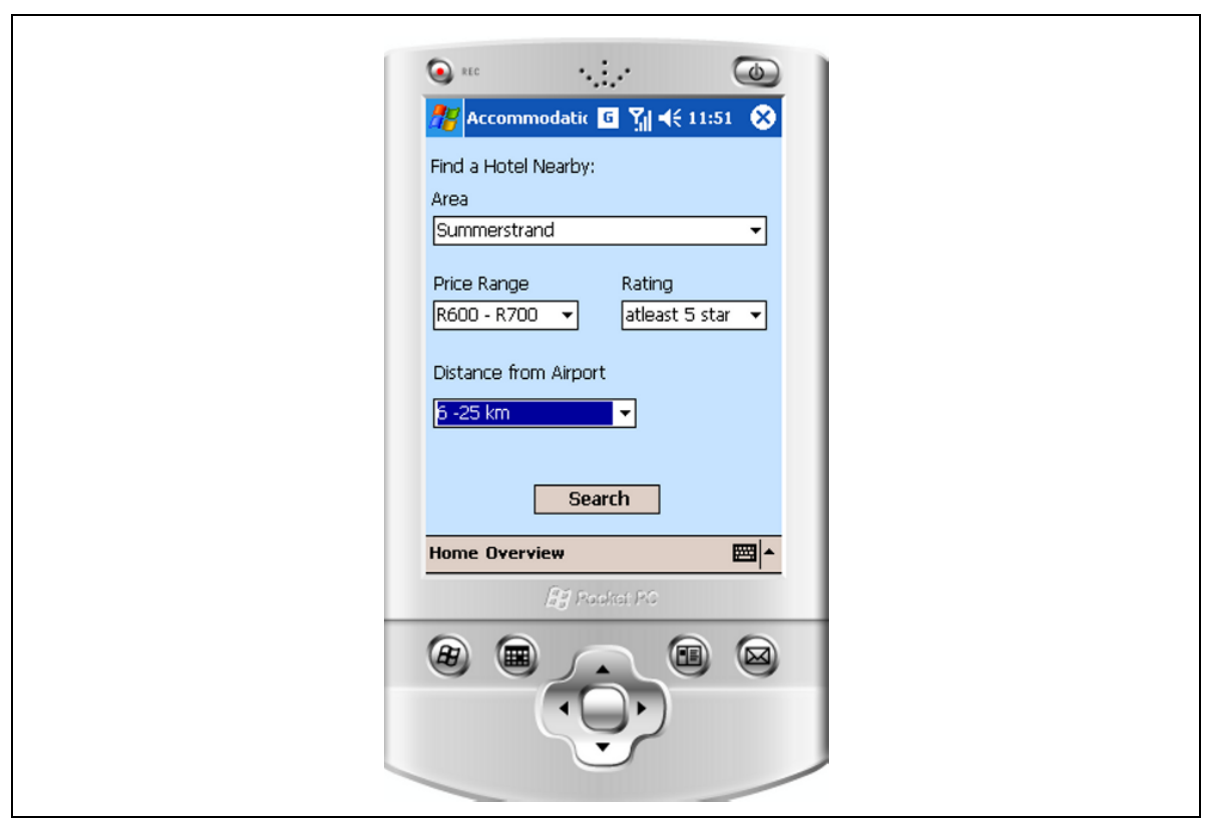

Fig. 5. Searching for accommodation in TravelSA

\subsection{Evaluation}

The students' prototypes were assessed in terms of usability and creativity, as outlined in Section 8.1. The usability of the prototypes was assessed by the author using a heuristic evaluation method together with Nielsen's ten heuristics [4]. Usability was determined in terms of the number of usability problems identified and the severity of these problems. The creativity of the designs was assessed according to the three creativity characteristics identified in Section 6, namely Novelty, Relevance (and effectiveness) and Germinal. The Novelty was assessed in terms of the degree of innovation shown; the Relevance in terms of the effectiveness of the solution in the specific context of use; and the Germinal in terms of the extension of existing interface metaphors and the development of new metaphors.

Both of the student examples presented in Sections 8.2 and 8.3 were evaluated as having a high degree of usability and creativity. Standard design aids, such as guidelines for mobile interface design were used, resulting in a high degree of usability (Section 2). Interface metaphors and iterative prototyping were used to develop the prototypes, resulting in a high degree of creativity (Sections 3 and 4). Existing metaphors were extended and adapted to a mobile context, exploiting the users' prior knowledge. Medical Mobile uses an Outlook metaphor to provide a mobile system for doctors; whilst TravelSA uses a map metaphor for selection of cities and areas of interest within a given city, and a search engine metaphor for finding accommodation within a city. These examples therefore provide evidence 
that the 'Usable Creativity' approach can be used to effectively teach creative interface design.

\section{Conclusions}

Insufficient time and attention is given in HCI curricula to encouraging creativity and innovation. Standard approaches to teaching interface design include the use of design aids such as guidelines and patterns. These approaches do not, however, encourage creativity. Design metaphors can offer several benefits for innovation provided that these are used correctly. Current approaches to teaching creative design encourage creativity but do not explicitly include the use of these design aids. This paper has proposed a combined approach, called 'Usable Creativity', which combines standard design aids with metaphors and prototyping to produce usable and creative design solutions. More research is needed, however, to develop evaluation methods and criteria to properly assess design creativity.

\section{References}

1. J. Preece, Y. Rogers, and H. Sharp, Interaction Design: Beyond Human-Computer Interaction (John Wiley \& Sons, Inc., 2002).

2. Microsoft Windows User Experience: Official Guidelines for User Interface Developers and Designers, Microsoft Professional Editions (Microsoft Press, 1999).

3. B. Shneiderman, Designing the User Interface: Strategies for Effective Human-Computer Interaction, Third Edition (Addison-Wesley, Reading, MA, 1997).

4. J. Nielsen, Ten Usability Heuristics, http://www.useit.com/papers/heuristic/heuristic_list.html, 1994.

5. IBM, Web design guidelines, (cited 12 June 2000); http://www-3.ibm/ibm/easy, 1999.

6. J. Gong and P. Tarasewich, Guidelines for Handheld Mobile Device Interface Design, in: Proceedings of DSI (Decision Sciences Institute) 2004 Annual Meeting, (Boston, Massechusetts, 2004), pp. 3751 - 3756.

7. M. van Welie and H. Traetteberg, Interaction Patterns in User Interfaces in: KoalaPLoP 2000 (Melbourne, Australia, 2000).

8. J.L. Wesson and N.L.O. Cowley, Designing with Patterns: Possibilities and Pitfalls, in: IFIP INTERACT'03 Workshop on Software \& Usability Cross-Pollination: The Role of Usability Patterns (Zurich, Switzerland, 2003).

9. N.L.O. Cowley and J.L. Wesson, An Experiment to Measure the Usefulness of Patterns in the Interaction Design Process, in: Human-Computer Interaction - INTERACT 2005, Lecture Notes in Computer Science 3585, (Springer, Berlin/Heidelberg, 2005), pp. 1142 1145.

10.A. Dix, T. Ormerod, M. Twidale, C. Sas, P.A.G. da Silva, and L. McKnight, Why bad ideas are a good idea, in: Inventivity: Teaching theory, design and innovation in HCI (HCIEd 2006) (Limerick, Ireland, 2006), pp. 9 - 14.

11.M. Lennon and L.J. Bannon, Worksheets in Practice: Gathering Artefacts for Reflection in Interaction Design Education, in: Inventivity: Teaching theory, design and innovation in HCI (HCIEd 2006) (Limerick, Ireland, 2006), pp. 29 - 34. 
12.M.K. Larusdottir, Using Rapid Contextual Design at Reykjavik University, in: Inventivity: Teaching theory, design and innovation in HCI (HCIEd 2006) (Limerick, Ireland, 2006), pp. $35-39$.

13.E. Frakjaer and K. Hornbaek, Metaphors of human thinking, in HCI: Habit, stream of thought, awareness, utterance and knowing, in: Human Factors and Ergonomics Society of Australia and the Australian Computer-Human Conference (HF2002/OzCHI2002) (Melbourne, Australia, 2002).

14.B.L.W. Wong, Inventivity in HCI Education, in: Inventivity: Teaching theory, design and innovation in HCI (HCIEd 2006) (Limerick, Ireland, 2006), pp. 67 - $72 .$.

15. S. Beckhaus, Seven Factors to Foster Creativity in University HCI Projects, in: Inventivity: Teaching theory, design and innovation in HCI (HCIEd 2006) (Limerick, Ireland, 2006), pp. $91-95$.

16. N. Jackson, Assessing Students' Creativity: Synthesis of Higher Education Teacher Views, http://www.heacademy.ac.uk/assets/York/documents/resources/resourcedatabase/id560_as sessing_creativity_synthesis_of_teachers_views.doc (The Higher Education Academy, 2005).

17. A. Sutcliffe, J. Karat, S. Bodker, and B. Gaver, Can We Measure Quality in Design or Do We Need To?, in: Proceedings of the 6th Conference on Designing Interactive Systems (University Park, Pennsylvania, USA, ACM Press, 2006), pp. 119 - 121. 